\title{
Porphyrie aiguë se manifestant sous forme de douleur abdominale durant la grossesse
}

\author{
Qazi Zain Sohail MD, Karima Khamisa MD
}

- Citation : CMAJ 2021 March 22;193:E419-22. doi : 10.1503/cmaj.202384-f

Voir la version anglaise de l'article ici : www.cmaj.ca/lookup/doi/10.1503/cmaj.202384

$\mathbf{U}$

ne femme blanche de 29 ans, ayant des antécédents d'hypothyroïdie et une résection intestinale partielle à distance pour appendice perforé, s'est présentée à notre hôpital; elle avait depuis quelques jours des crampes soudaines, des douleurs abdominales basses diffuses et non irradiantes, des nausées, des vomissements et des diarrhées aqueuses. Après confirmation d'une grossesse intra-utérine de 4 semaines, elle a reçu son congé du service des urgences avec un diagnostic de nausées et vomissements de la grossesse (nausées matinales). Cependant, à sa troisième présentation sur 2 jours avec des symptômes progressifs, elle a été admise pour l'administration d'analgésiques et de solutions par voie parentérale.

La patiente présentait d'excellentes capacités fonctionnelles avant cet épisode aigu et n'avait aucun antécédent de douleurs abdominales récurrentes, d'allergies, de prise de médicaments ou de suppléments, de consommation de drogues à usage récréatif, d'exposition à des métaux lourds, de voyages récents ou de contacts avec des malades. Son partenaire a confirmé cette information. La revue des systèmes n'a pas évoqué d'autres causes des douleurs abdominales aiguës (tableau 1)1. À l'examen, la patiente était afébrile, avec une tension artérielle de 144/87 $\mathrm{mm} \mathrm{Hg}$ et une fréquence cardiaque de 82 battements par minute; sa saturation en oxygène était de $100 \%$ par oxymétrie de pouls à l'air ambiant. Elle présentait une sensibilité diffuse de l'abdomen, et les résultats de ses examens neurologiques, cardiovasculaires, respiratoires et cutanés étaient sans particularité.

Au cours des jours suivants, sa douleur s'est aggravée progressivement et elle a dû recevoir jusqu'à $20 \mathrm{mg}$ d'hydromorphone par jour au moyen d'un dispositif d'administration continue ambulatoire. Elle est également devenue hypertensive et a décrit des hésitations urinaires; nous l'avons traitée avec du labétalol pour contrôler la tension artérielle, et avec de la pyridoxine-doxylamine, du dimenhydrinate et du métoclopramide pour les nausées.

Les résultats des examens de laboratoire étaient pour la plupart dans les limites de la normale, à l'exception d'une

\section{POINTS CLÉS}

- Les porphyries aiguës sont des troubles génétiques rares qui se manifestent par des symptômes neurologiques et systémiques, notamment des douleurs abdominales, une hyponatrémie et des crises.

- En cas de suspicion de porphyrie, un dépistage urinaire doit être effectué.

- Si un dépistage urinaire de la porphyrie est positif, le traitement de la porphyrie aiguë ne doit pas être retardé.

- L'identification et l'évitement des déclencheurs, une alimentation riche en glucides, l'administration d'hémine par voie intraveineuse et des soins de soutien sont les principaux traitements de la porphyrie aiguë.

légère acidose métabolique probablement secondaire à une cétose de famine (avec une glycémie normale). La patiente présentait une hyponatrémie compatible avec un syndrome de sécrétion inappropriée d'hormone antidiurétique (SIADH), et son taux d'aminotransférase (ALT) était légèrement élevé (tableau 2). La radiographie du thorax était normale, et l'électrocardiogramme montrait un rythme sinusal normal avec une possible arythmie sinusale. Compte tenu de ses antécédents chirurgicaux, nous avons demandé une échographie abdominale et des examens d'imagerie par résonance magnétique qui, à part un simple hydrosalpinx gauche, n'ont révélé aucune pathologie des voies hépatobiliaires, génitourinaires ou gastro-intestinales. L'échographie Doppler de la veine porte n'a pas montré de thrombus pouvant expliquer l'élévation de l'ALT.

Compte tenu de la numération leucocytaire normale de la patiente, de l'absence de fièvre, des écouvillons cervicaux négatifs pour la chlamydia et la gonorrhée, de l'écouvillon nasopharyngé négatif pour la maladie à coronavirus 2019, et de l'analyse et de la culture d'urine normales, nous avons exclu une cause infectieuse pour ses douleurs abdominales et ses nausées. Un taux normal de protéine $C$ réactive et une imagerie abdominale plaidaient en défaveur d'une maladie 
Tableau 1 : Considérations pour le diagnostic différentiel des douleurs abdominales aiguës ${ }^{1}$

\begin{tabular}{|c|c|}
\hline Chirurgie & $\begin{array}{l}\text { - Occlusion intestinale } \\
\text { - Viscères perforés } \\
\text { - Colite ischémique } \\
\text { - Pathologie de l'arbre biliaire } \\
\text { - Pancréatite } \\
\text { - Obstruction urétérale } \\
\text { - Anévrisme de l'aorte abdominale } \\
\text { - Torsion ovarienne } \\
\text { - Trauma }\end{array}$ \\
\hline $\begin{array}{l}\text { Drogues et } \\
\text { toxines }\end{array}$ & $\begin{array}{l}\text { - Consommation de cocaïne } \\
\text { - Saturnisme }\end{array}$ \\
\hline $\begin{array}{l}\text { Maladies } \\
\text { infectieuses }\end{array}$ & $\begin{array}{l}\text { - Gastroentérite } \\
\text { - Hépatite } \\
\text { - Infection génito-urinaire } \\
\text { - Zona }\end{array}$ \\
\hline $\begin{array}{l}\text { Enjeux } \\
\text { métaboliques }\end{array}$ & $\begin{array}{l}\text { - Maladies inflammatoires de l'intestin } \\
\text { - Acidocétose diabétique } \\
\text { - Hypercalcémie } \\
\text { - Insuffisance surrénale } \\
\text { - Porphyrie aiguë }\end{array}$ \\
\hline $\begin{array}{l}\text { Douleur } \\
\text { référée }\end{array}$ & $\begin{array}{l}\text { - Cardiaque : infarctus du myocarde, péricardite } \\
\text { pulmonaire : pneumonie, embolie pulmonaire, } \\
\text { pneumothorax) } \\
\text { - Esophagienne : spasme œsophagien, reflux } \\
\text { gastrique } \\
\text { - Musculosquelettique : comme la douleur } \\
\text { thoracique } \\
\text { - Compression de la moelle épinière ou des } \\
\text { racines nerveuses } \\
\text { - Torsion testiculaire }\end{array}$ \\
\hline Autres & $\begin{array}{l}\text { - Syndrome du côlon irritable } \\
\text { - Intolérance au lactose } \\
\text { - Douleur abdominale fonctionnelle } \\
\text { - Troubles psychiatriques } \\
\text { - Fièvre méditerranéenne familiale } \\
\text { - Déficit en inhibiteur de l'estérase } \mathrm{C} 1\end{array}$ \\
\hline
\end{tabular}

inflammatoire de l'intestin et d'autres affections autoimmunes ${ }^{1}$. En raison des antécédents chirurgicaux de la patiente, notre diagnostic de travail était alors une douleur médiée par la présence d'une cicatrice sur utérus gravide.

Cependant, étant donné les douleurs abdominales persistantes et intraitables, l'hésitation urinaire, l'hypertension suggérant une dysrégulation autonome et le SIADH, nous avons envisagé la possibilité d'une porphyrie et demandé un dépistage urinaire, qui s'est révélé positif le dixième jour (tableau 2). Nous avons diagnostiqué une porphyrie aiguë, probablement déclenchée par la grossesse. L'analyse quantitative du même échantillon d'urine a permis d'observer un
Tableau 2 : Sommaire des résultats d'examens de laboratoire de la patiente

\begin{tabular}{|c|c|}
\hline Analyse & Résultat (valeurs normales) \\
\hline Hémoglobine (g/L) & $149(115-155)$ \\
\hline Volume corpusculaire moyen (fL) & $83,9(80-100)$ \\
\hline Globules blancs (× 109/L) & $7,5(3,5-10,5)$ \\
\hline Thrombocytes (× 109/L) & $184(130-380)$ \\
\hline Sodium (mmol/L) & $139(136-144)$ \\
\hline Potassium (mmol/L) & $4,1(3,5-5,1)$ \\
\hline Créatinine ( $\mu \mathrm{mol} / \mathrm{L})$ & $49(49-84)$ \\
\hline Bicarbonate (mmol/L) & $20(19-30)$ \\
\hline Calcium (mmol/L) & $2,42(2,24-2,58)$ \\
\hline Albumine (g/L) & $46(36-47)$ \\
\hline Aspartate aminotransférase (UI/L) & $24(2-25)$ \\
\hline Alanine aminotransférase (UI/L) & $31(6-30)$ \\
\hline Phosphatase alcaline (UI/L) & $49(46-118)$ \\
\hline Bilirubine totale $(\mu \mathrm{mol} / \mathrm{L})$ & $18(\leq 11)$ \\
\hline Lactate (mmol/L) & $1,2(0,25-2,5)$ \\
\hline Lipase (UI/L) & $31(14-85)$ \\
\hline $\begin{array}{l}\text { Gonadotrophine chorionique } \\
\text { humaine }(\mathrm{mUl} / \mathrm{mL})\end{array}$ & $1672(\leq 5)$ \\
\hline Sodium dans l'urine (mEq/L) & 172 \\
\hline Osmolalité (mOsm/kg) & $269(280-295)$ \\
\hline Osmolalité urinaire (mOsm/kg) & $794(50-1200)$ \\
\hline Protéines C réactives (mg/L) & $0,4(<10)$ \\
\hline Thyréostimuline (mUI/L) & $1,88(0,27-4,2)$ \\
\hline $\begin{array}{l}\text { Dépistage de la porphyrie dans } \\
\text { l'urine }\end{array}$ & Positif \\
\hline $\begin{array}{l}\text { Analyse quantitative } \\
\text { des porphyrines }\end{array}$ & \\
\hline $\begin{array}{l}\text { Porphobilinogène désaminase } \\
\text { sérique }(\mu \mathrm{mol} / \mathrm{L})\end{array}$ & $27(29-49)$ \\
\hline $\begin{array}{l}\text { Rapport porphobilinogène/ } \\
\text { créatinine urinaire } \\
(\mu \mathrm{mol} / \mathrm{mmol} \mathrm{Cr})\end{array}$ & $4,3(<0,8)$ \\
\hline $\begin{array}{l}\text { Rapport acide aminolévulinique/ } \\
\text { créatinine urinaire } \\
(\mu \mathrm{mol} / \mathrm{mmol} \mathrm{Cr})\end{array}$ & $14,1(<5)$ \\
\hline $\begin{array}{l}\text { Analyse quantitative de la } \\
\text { porphyrine urinaire } \\
(\mathrm{nmol} / \mathrm{mmol} \mathrm{Cr})\end{array}$ & $\begin{array}{l}\text { - Uroporphyrine I : 204,6 } \\
\text { (élevée) } \\
\text { - Uroporphyrine III : 34,4 } \\
\text { (élevée) } \\
\text { - Coproporphyrine I : 316,8 } \\
\text { (élevée) } \\
\text { - Coproporphyrine III : 1113,6 } \\
\text { (élevée) }\end{array}$ \\
\hline $\begin{array}{l}\text { Analyse quantitative de la } \\
\text { porphyrine fécale (nmol/g) }\end{array}$ & $\begin{array}{l}\text { - Coproporphyrine III : 2376,1 } \\
\text { ( } \leq 12) \\
\text { - Coproporphyrine I : 252,2 } \\
\text { ( } \leq 13) \\
\text { - Protoporphyrine : } 6,5(\leq 38)\end{array}$ \\
\hline
\end{tabular}


rapport porphobilinogène-créatinine urinaire élevé. L'analyse complète des porphyrines dans les urines et les selles a révélé des taux de coproporphyrine sensiblement élevés avec une protoporphyrine normale, ce qui a confirmé le diagnostic de coproporphyrie héréditaire.

En raison de la gravité de la crise, nous avons décidé de mettre en place une perfusion de produit à base d'hémine obtenu de la Société canadienne du sang (Panhematin) qui corrige la carence en hème dans le foie et régule à la baisse les précurseurs de porphyrine à l'origine des crises aiguës ${ }^{2}$. Après avoir consulté le site Internet de l'American Porphyria Foundation, nous avons cessé d'administrer la pyridoxine-doxylamine, le dimenhydrinate et le métoclopramide, qui peuvent exacerber les épisodes de porphyrie aiguë3. Nous avons également prescrit une charge élevée de glucides. Les symptômes de la patiente se sont améliorés après 4 jours de perfusion d'hémine et elle a été renvoyée chez elle avec une faible dose d'hydromorphone, qui a été interrompue peu après.

Lors du suivi à 1 mois, les symptômes de nausée de la patiente s'étaient améliorés, sa douleur était bien contrôlée avec une analgésie minimale, et sa tension artérielle et son taux de sodium plasmatique s'étaient normalisés. La famille de la patiente a confirmé que sa grand-mère paternelle avait signalé des épisodes intermittents d'urine violette. La patiente et son père ont finalement été testés positifs pour la mutation génique responsable de la coproporphyrie héréditaire, bien que son père reste asymptomatique. En préparation du travail et de l'accouchement, nous avons surveillé la réapparition de tous les symptômes et signes cliniques de la porphyrie aiguë et évité l'hypoglycémie et les médicaments connus pour déclencher des crises. La patiente a accouché d'un bébé en bonne santé.

\section{Discussion}

\section{Profil épidémiologique}

Le terme "porphyrie aiguë » désigne un groupe de maladies génétiques rares caractérisées par diverses déficiences enzymatiques dans la voie de biosynthèse de l'hème $e^{4,5}$. Le sous-type le plus courant de porphyrie aiguë est la porphyrie aiguë intermittente; les autres comprennent la porphyrie variegata, la coproporphyrie héréditaire et la très rare porphyrie par déficit en acide aminolévulinique déshydratase $e^{4,5}$. Les porphyries symptomatiques combinées ont une prévalence estimée à 10 par million dans les populations européennes, la porphyrie aiguë intermittente étant environ 2 fois plus fréquente que la porphyrie variegata et 6 fois plus fréquente que la coproporphyrie héréditaire ${ }^{6}$. Ces affections sont autosomiques dominantes, avec une pénétrance incomplète, et, par conséquent, les porteurs d'une mutation génique peuvent ne jamais avoir de crises aiguës ${ }^{6}$. Les symptômes se manifestent généralement chez les jeunes femmes entre la deuxième et la quatrième décennie de leur vie $e^{5,7}$.

\section{Manifestations cliniques}

Les symptômes et signes habituels des crises aiguës comprennent des douleurs abdominales diffuses (jusqu'à $90 \%$ des patients), des nausées, des vomissements ou des modifications des habitudes d'évacuation intestinale, une urine qui devient foncée lorsqu'elle est exposée à la lumière et une hyponatrémie due à une sécrétion inappropriée d'hormone antidiurétique ou à une perte de sel rénale pouvant précipiter les crises $^{5}$. Les patients peuvent également présenter des symptômes neuropsychiatriques tels que des troubles de l'humeur, une psychose, des modifications du comportement, un dysfonctionnement autonome, une neuropathie périphérique sensorielle ou motrice, voire une détresse respiratoire ${ }^{5}$. Des lésions cutanées bulleuses peuvent être associées à une porphyrie variegata (50\% des patients) et à une coproporphyrie héréditaire $(<20 \%$ des patients $)^{5}$. Un rapport de cas décrit un syndrome d'encéphalopathie postérieure réversible chez une patiente en post-partum présentant une porphyrie aiguë intermittente connue ${ }^{8}$.

\section{Diagnostic}

Compte tenu de sa rareté et de son profil de présentation, il est difficile de diagnostiquer la porphyrie aiguë. Les patients peuvent subir de multiples interventions chirurgicales abdominales; l'étude d'une série de cas a révélé un retard diagnostique moyen de $10 \mathrm{ans}^{7,9}$. Le diagnostic de la porphyrie aiguë nécessite la détection d'une augmentation du porphobilinogène urinaire ${ }^{5}$. Le dépistage initial des porphyrines urinaires peut être réalisé plus rapidement que la quantification définitive, bien que, en présence de symptômes typiques et en l'absence de causes secondaires, le dépistage puisse être suffisant pour amorcer un traitement $^{5}$. La quantification des porphyrines fécales ou plasmatiques est nécessaire pour sous-typer l'affection ${ }^{5}$. Des études génétiques doivent être demandées chez les patients présentant une élévation confirmée des taux de porphyrines, ainsi que chez les parents au premier degré de ces patients ${ }^{5}$.

Divers déclencheurs sont associés aux crises de porphyrie aiguë, notamment la prise de médicaments sur ordonnance, comme des antibiotiques, des contraceptifs oraux et des anticonvulsivants, ainsi que l'usage de drogues à des fins récréatives et une forte consommation d'alcool ${ }^{5}$. Des bases de données exhaustives sur les médicaments ont été créées pour aider les prestataires de soins de santé et les patients souffrant de ces troubles à faire des choix éclairés ${ }^{3}$. Les changements physiologiques des concentrations hormonales - causés notamment par une grossesse, une diminution de l'apport alimentaire, le stress ou des infections sont des déclencheurs connus ${ }^{5}$.

Les causes secondaires de la porphyrinurie doivent toujours être envisagées, notamment les maladies du foie aiguës ou chroniques, les troubles de l'hémoglobine tels que la thalassémie ou la drépanocytose, et l'exposition à des toxines environnementales comme le plomb, le mercure ou l'arsenic ${ }^{10}$. On doit accorder une importance particulière à la toxicité du plomb, car celle-ci peut reproduire les symptômes de la porphyrie aiguë sur le plan clinique. 


\section{Prise en charge}

Dans les crises aiguës, une analyse approfondie des antécédents médicaux du patient peut permettre d'identifier des déclencheurs ${ }^{5}$. Une analgésie adéquate est cruciale et, dans certains cas, une analgésie contrôlée par le patient peut être privilégiée $^{5}$. Il faut procéder à une évaluation minutieuse des électrolytes urinaires et du statut volumique, notamment pour évaluer l'hyponatrémie. Les glucides peuvent inhiber la voie intrinsèque de biosynthèse de l'hème, et une augmentation de l'apport à court terme $(300-500 \mathrm{~g} / \mathrm{j})$ peut suffire pour traiter les crises légères ${ }^{5}$. Les diététiciens peuvent aider à assurer un apport adéquat en glucides.

Chez les patients qui présentent des symptômes neurologiques, une hyponatrémie ou une instabilité clinique, le traitement repose sur l'administration d'hémine par voie intraveineuse ${ }^{5}$. Au Canada, l'hémine n'est disponible que par l'intermédiaire de la Société canadienne du sang². Bien que son efficacité n'ait pas été prouvée au moyen d'essais randomisés, on considère qu'elle soulage les symptômes plus rapidement et réduit le risque de complications ${ }^{5}$. L'hémine a été utilisée avec succès chez les patientes enceintes, et la combinaison d'hémine, de glucose et de sulfate de magnésium s'est avérée une stratégie de traitement efficace pour le syndrome d'encéphalopathie réversible postérieure du post-partum ${ }^{8}$. Les benzodiazépines peuvent également être indiquées pour les crises aiguës ${ }^{5}$. La protection solaire est recommandée pour ces patients, étant donné leur prédisposition à la photosensibilité ${ }^{5}$. Bien que les crises récurrentes soient rares, des perfusions prophylactiques d'hémine peuvent être envisagées.

À long terme, les patients atteints de porphyrie aiguë peuvent développer des complications, notamment une atteinte neurologique ou des maladies rénales chroniques, et ils présentent un risque accru de carcinome hépatocellulaire ${ }^{5}$. Dans de rares cas, la greffe de foie peut être la seule option curative $^{5}$. Les données sur le pronostic sont rares et, bien que les crises soient rarement fatales, les experts plaident pour un dépistage et une prise en charge précoces afin de réduire les complications ${ }^{5}$.

\section{Références}

1. Stephenson M. Non-surgical causes of abdominal pain. BMJ 2008;336(Suppl S6):080246.

2. Verbrugghe S, Ali S, Lee K. Hemin for injection (panhematin): budget impact analysis. CADTH Technology Review no 24. Ottawa: Canadian Agency For Drugs And Technologies In Health (CADTH); 2019.

3. Porphyria. Bethesda (MD): American Porphyria Foundation. Accessible ici : https://porphyriafoundation.org/for-healthcare-professionals/porphyria/ (consulté le 15 juin 2020).

4. Anderson KE, Bloomer JR, Bonkovsky HL, et al. Recommendations for the diagnosis and treatment of the acute porphyrias. Ann Intern Med 2005;142:439-50.

5. Stein PE, Badminton MN, Rees DC. Update review of the acute porphyrias. Br J Haematol 2017;176:527-38.

6. Elder $\mathrm{G}$, Harper $\mathrm{P}$, Badminton $\mathrm{M}$, et al. The incidence of inherited porphyrias in Europe. J Inherit Metab Dis 2013;36:849-57.

7. Bonkovsky HL, Maddukuri VC, Yazici C, et al. Acute porphyrias in the USA: features of 108 subjects from porphyrias consortium. Am J Med 2014;127:1233-41.

8. Zhang J, Hu Y, Zheng J, et al. Treatment of acute intermittent porphyria during pregnancy and posterior reversible encephalopathy syndrome after delivery: a case report. Exp Ther Med 2017;14:5554-6.

9. Indika NLR, Kesavan T, Dilanthi HW, et al. Many pitfalls in diagnosis of acute intermittent porphyria: a case report. BMC Res Notes 2018;11:552.

10. Barber T, Jacyna M. Acute lead intoxication from medications purchased online presenting with recurrent abdominal pain and encephalopathy. J R Soc Med 2011;104:120-3.

Intérêts concurrents : Aucun déclaré

Cet article a été révisé par des pairs.

Les auteurs ont obtenu le consentement de la patiente.

Affiliations : Faculté de médecine, Université d'Ottawa, Ottawa, Ont.

Collaborateurs : Qazi Sohail a contribué à l'élaboration et à la conception de l'étude et a rédigé le manuscrit. Les deux auteurs ont révisé de façon critique le contenu intellectuel important du manuscrit; ils ont donné leur approbation finale pour la version destinée à être publiée et assument l'entière responsabilité de tous les aspects du travail.

Propriété intellectuelle du contenu : Il s'agit d'un article en libre accès distribué conformément aux modalités de la licence Creative Commons Attributions (CC BY-NC-ND 4.0), qui permet l'utilisation, la diffusion et la reproduction dans tout médium à la condition que la publication originale soit adéquatement citée, que l'utilisation se fasse à des fins non commerciales (c.-à-d. recherche ou éducation) et qu'aucune modification ni adaptation n'y soit apportée. Voir : https://creativecommons.org/licenses/by-nc-nd/4.0/deed.fr.

Correspondance : Karima Khamisa, kkhamisa@toh.ca 